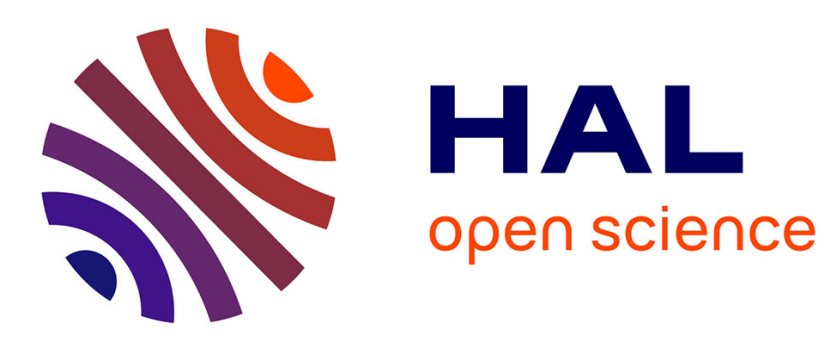

\title{
Edmund Stoner and white dwarf stars
}

Edwin Thomas

\section{To cite this version:}

Edwin Thomas. Edmund Stoner and white dwarf stars. Philosophical Magazine, 2011, pp.1. 10.1080/14786435.2011.586377 . hal-00710060

\section{HAL Id: hal-00710060 https://hal.science/hal-00710060}

Submitted on 20 Jun 2012

HAL is a multi-disciplinary open access archive for the deposit and dissemination of scientific research documents, whether they are published or not. The documents may come from teaching and research institutions in France or abroad, or from public or private research centers.
L'archive ouverte pluridisciplinaire HAL, est destinée au dépôt et à la diffusion de documents scientifiques de niveau recherche, publiés ou non, émanant des établissements d'enseignement et de recherche français ou étrangers, des laboratoires publics ou privés. 


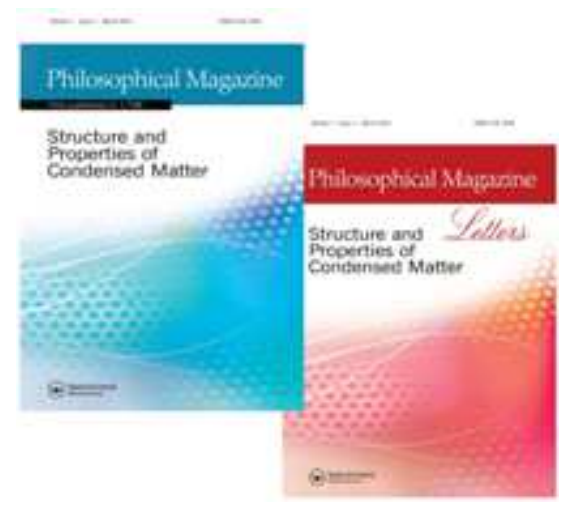

\section{Edmund Stoner and white dwarf stars}

\begin{tabular}{|r|l|}
\hline Journal: & Philosophical Magazine \& Philosophical Magazine Letters \\
\hline Manuscript ID: & TPHM-11-Mar-0088.R1 \\
\hline Journal Selection: & Philosophical Magazine \\
\hline Date Submitted by the \\
Author: & 13-Apr-2011 \\
\hline Complete List of Authors: & Thomas, Edwin; University of Leicester, Physics and Astronomy \\
\hline Keywords: & total energy methods, electron theory, electron energy states \\
\hline Keywords (user supplied): & cosmology, white dwarfs, Chandrasekhar mass \\
\hline &
\end{tabular}

\section{SCHOLARONE ${ }^{\text {TM }}$} Manuscripts 


\title{
Edmund Stoner and white dwarf stars
}

\author{
E. Thomas, Department of Physics and Astronomy, University of \\ Leicester, University Road, Leicester LE1 7RH, UK
}

\begin{abstract}
The discovery of a limiting mass for white dwarf stars is today usually attributed to Subramanian Chandrasekhar. However it appears that an article by Edmund Stoner, which appeared in the Philosophical Magazine in 1930, was the first publication to give a convincing demonstration of the existence of a limiting mass for white dwarfs. We examine here why it is that the contributions of Stoner and others towards this discovery have been largely forgotten.
\end{abstract}

\section{Introduction}

The Biographical Memoires of Fellows of the Royal Society [1] tell us that Edmund Clifton Stoner was born in Esher in the county of Surrey in 1899. When he was in his teens his parents moved to Bolton in Lancashire where he won a scholarship that enabled him to attend Bolton Grammar School. From there he entered Cambridge University in 1918 graduating in 1921 with a first-class degree in the Natural Sciences Tripos. Surprisingly, although the physics lectures given to undergraduates were quite mathematical in content there was no mathematics teaching in the syllabus, students being expected to pick up what they needed on their own. Stoner was conscious of this lack of formal mathematics training throughout his career.

Between 1921 and 1924 Stoner worked for a PhD in the Cavendish laboratory with Ernest Rutherford as his official supervisor. His research topic was the measurement of the absorption of X-rays by a selected group of elements. He obtained his PhD in 1924, despite a prolonged period in hospital after being diagnosed with diabetes. Towards the end of his time at the Cavendish he wrote a paper entitled The distribution of electrons among atomic energy levels. This paper, published in the Philosophical Magazine [2] preceded Pauli's enunciation of his exclusion principle and played a significant role in its creation: in effect it contained an explicit statement of the exclusion principle. It should be noted that the exclusion principle was to play a crucial role in the theory of white dwarfs.

After deciding that he did not wish to remain in Cambridge, Stoner successfully applied for a lectureship in the Physics Department of the University of Leeds. There, where he remained until his retirement in 1963 , he switched to research in theoretical physics and 
embarked on pioneering work to understand the magnetic properties of matter: this is the work for which he is best known. His interest in white dwarfs was sparked by Fowler's paper On Dense Matter [3]. Over the period 1929 to 1932 he published the pioneering papers on white dwarfs, which are the subject of this article. In particular he deduced the existence of a limiting mass for white dwarfs now known as the Chandrasekhar mass.

\section{White dwarfs}

White dwarfs are hot, very compact, stars that have ceased nuclear burning. Their high temperature and low luminosity places them in the bottom left-hand corner of the Hertzprung- Russell diagram [4]. The white dwarf, 40 Eridani B, recorded by William Herschel in 1783 was the first to be detected [5]. By the late 1920s, when Stoner started his investigations, the masses, luminosities and surface temperatures of a small number of white dwarfs had been measured. The best-known example, Sirius $B$, is the faint companion of Sirius, the brightest star in the night sky. The observational data at this time for Sirius $B$ gave its mass to be 0.85 solar masses, its surface temperature $8000 \mathrm{~K}$ and its radius 21000 $\mathrm{km}$. These figures imply a mean density of about $5 \times 10^{7} \mathrm{~kg} \mathrm{~m}^{-3}$. At the time this density exceeded that of any other known object by several orders of magnitude. For example the mean density of the Sun is about $1400 \mathrm{~kg} \mathrm{~m}^{-3}$ and the density of osmium metal, the densest element, is $2.2 \times 10^{4} \mathrm{~kg} \mathrm{~m}^{-3}$. It is interesting to note that, with these values for the mass and radius of Sirius $B$, the General Theory of Relativity predicts that light coming from its surface would have a red shift of $20 \mathrm{~km} \mathrm{~s}^{-1}$. A measurement of this red shift published by W S Adams [6] produced a value of $19 \mathrm{~km} \mathrm{~s}^{-1}$. However, we now know that this apparent agreement between The General Theory of Relativity and the data on Sirius B, which so delighted Eddington, is coincidental. Adam's measurement of redshift is too low by a factor of 4 and the radius is too large by a factor of almost 4 . The modern values for the mass, temperature, radius and red shift of Sirius B are $0.98 M_{\text {solar }}, 22000 \mathrm{~K}$ and $6000 \mathrm{~km}$ respectively and the red shift, measured using the Hubble space telescope, is $80 \mathrm{~km} \mathrm{~s}^{-1}$ [7]. With these measurements the density of Sirius B becomes $2 \times 10^{9} \mathrm{~kg} \mathrm{~m}^{-3}$ or 2 tonnes $\mathrm{cm}^{-3}$.

Such high densities seemed at the time to raise a paradox: matter compressed to this degree must be completely ionised so what happens to the star as its reaches the end of its lifetime and starts to cool? In the words of Sir Arthur Eddington [8]

'The star could not stop losing heat, but it would not have enough energy to cool down'.

Eddington supposed that for the star to reach a stable cold state, its nuclei and electrons needed to recombine into atoms. But to do this the star needed to regain normal density by expanding against the force of gravity, which it could not do. According to classical physics, a 
cold ionised gas cannot exert any pressure so the star was doomed to continue contracting indefinitely, a situation that Eddington regarded as absurd.

The resolution of this paradox was provided by the newly discovered quantum mechanics and the Pauli Exclusion Principle. The Cambridge theoretician R H Fowler realised that even at the absolute zero of temperature a very dense electron gas will exert a large pressure and that this pressure could support a white dwarf star against collapse [3]. Fowler assumed that the electrons occupied the lowest energy states allowed by the Pauli Principle, which states that only two electrons, one in each of two spin states, can occupy a state of volume $h^{3}$ in six-dimensional phase space, where $h$ is the Planck constant. Such a gas is said to be degenerate; the uppermost filled state has a momentum $p_{0}$ which, as a function of number density of electrons $n$, is given, at absolute zero, by

$$
p_{0}=h\left(\frac{3}{8 \pi}\right)^{1 / 3} n^{1 / 3} \text {. }
$$

So a high density of electrons in physical space implies that they have high momenta and hence that they exert a large pressure which, for non-relativistic electrons, is given as a function of the mass density $\rho=\mu_{e} m_{H} n$ by the expression

$$
P=\frac{h^{2}}{5 m_{e}}\left(\frac{3}{8 \pi}\right)^{2 / 3}\left(\frac{\rho}{\mu_{e} m_{H}}\right)^{5 / 3}
$$

where $m_{e}$ is the mass of an electron, $m_{H}$ the mass of hydrogen and $\mu_{e}$ the average atomic weight per electron. At the time, white dwarfs, being highly evolved stars, were thought to be composed of heavy elements so the value of $\mu_{e}$ was taken to be about 2.5 . We now know that white dwarfs are composed mainly of carbon and oxygen so the correct value of $\mu_{e}$ is very close to 2 . The nuclei being much more massive than the electrons do not contribute significantly to the pressure.

\section{Stoner's papers on white dwarfs}

In the first of three papers that appeared in the Philosophical Magazine [9], Stoner took Fowler's idea that white dwarfs are supported by electron degeneracy pressure and asked the question: is there a limit to the density of such stars? He modelled a star as a sphere of ionised gas having uniform density at zero temperature. Then, from the kinetic energy $E_{K}$ of the electrons and the gravitational binding energy $E_{G}$ of the star, the density is obtained by minimising the total energy with respect to the number density $n$, that is he imposed the condition 


$$
\frac{d}{d n}\left(E_{K}+E_{G}\right)=0
$$

From this condition he obtained the following expression for the mean mass density of a star of mass $M$ :

$$
\rho=3.85 \times 10^{9}\left(\frac{M}{M_{\text {solar }}}\right)^{2} \mathrm{~kg} \mathrm{~m}^{-3} .
$$

Note that this expression gives a value for the mean density of Sirius B in fairly good agreement with the modern value but not in good agreement with the 1930's value. Note also that there is no suggestion of a limiting mass with Fowler's non-relativistic equation (equation 2).

Following the appearance of Stoner's paper, Wilhelm Anderson, a German physicist working at Tartu University in Estonia, noticed that for masses beyond about a solar mass the electron energies become relativistic [10]. So Fowler's equation of state, which is derived using Newtonian Mechanics, will not be valid for the more massive stars. Anderson derived a relativistic equation of state and, despite his derivation being not strictly correct, deduced from it that there is a limiting mass for white dwarfs of $0.68 M_{\text {solar }}$; with the modern value $\mu_{e}=2$ this becomes $1.1 M_{\text {solar }}$.

Stoner responded to Anderson in a second paper [11] (reproduced as a facsimile following this commentary) in which he pointed out the error in Anderson's derivation of the relativistic equation of state and derived the correct equation - Stoner's equation is now known as the Stoner-Anderson equation. In the extreme relativistic limit this equation of state tends to the simple form

$$
P=\frac{c h}{4}\left(\frac{3}{8 \pi}\right)^{1 / 3}\left(\frac{\rho}{\mu_{e} m_{H}}\right)^{4 / 3}
$$

Stoner used his energy minimisation method with the constant density approximation and his relativistic equation of state to obtain the mass of a star for any value of the electron density $n$, or, equivalently, $\rho$. He found that as the density increases, the mass of the star increases more and more slowly, eventually reaching a limiting value given by the expression

$$
M_{L}=\frac{3}{16 \pi}\left(\frac{5 h c}{2 G}\right)^{3 / 2} \frac{1}{\left(\mu_{e} m_{H}\right)^{2}}
$$

With $\mu_{e}=2$ this gives $M_{\mathrm{L}}=1.56 M_{\text {solar, }}$ which should be compared with the more accurate value of $1.44 M_{\text {solar }}$ obtained by numerical integration of the equations of stellar structure. It is interesting to note that Stoner did not write out the expression above for $M_{L}$ explicitly, so 
missed the significant observation that $M_{\mathrm{L}}$ is proportional to the Planck mass $\left(\frac{h c}{G}\right)^{1 / 2}$ cubed.

The Planck mass occurs whenever gravity, quantum mechanics and relativity play an essential role; for example see [12].

We can understand the reason for the existence of a limiting mass using the constant energy approximation as follows. With the non-relativistic equation of state [equation (2)], as the star shrinks the kinetic energy $E_{\mathrm{K}}$ increases faster than the potential energy $E_{\mathrm{G}}$ so there is always an equilibrium radius. But with the limiting form of the equation of state [equation (5)], $E_{\mathrm{K}}$ becomes proportional to $N^{4 / 3} / R$, where $R$ is the radius of the star and $N$ the number of electrons. As the gravitational energy $E_{\mathrm{G}}$ is proportional to $N^{2} / R$, both $E_{\mathrm{K}}$ and $E_{\mathrm{G}}$ increase at the same rate as the star contracts. So if $\left|E_{G}\right|>E_{K}$ the star will collapse and if $\left|E_{G}\right|<E_{K}$ the star will expand until the dependence of $E_{\mathrm{K}}$ on $R$ alters allowing equilibrium to be reached. The limiting mass will occur when the two terms are equal. This condition determines the value of $N$ and hence the limiting mass of the star.

Stoner also looked for and failed to find a relationship between the measured masses and luminosities of the few known white dwarfs. We now know that there is no relationship because white dwarfs have ceased nuclear burning and are cooling down, so the luminosity of a white dwarf depends on its age.

Stoner's third paper [13] written in collaboration with his research student Frank Tyler is a sequel to his two previous papers. In it he attempts to obtain the actual density distribution in order to determine to what extent his previous results based on the constant density approximation would need to be modified. In his own words:

'The problem has proved more intractable than was anticipated, and a completely satisfactory solution has not been reached'.

He also addressed the fate of a star whose mass lay above the limit. He concluded that the star would contract, heat up and gradually lose mass through radiation until its mass dropped below the limiting value, allowing it to stabilise. It should be born in mind that, in the early 1930s, nuclear physics was in its infancy so his conclusions were reasonable given the state of knowledge at the time. We now know that, rather than a slow contraction, a catastrophic collapse would occur initiated by the onset of the inverse beta decay reaction $e^{-}+p \rightarrow n+v_{e}$, which undermines the support of the star by removing electrons. The collapse gives rise to very rapid heating, causing thermonuclear burning of the carbon and oxygen. The resultant release of energy is rapid enough to blow the star apart - an event now identified as a supernova of type $1 \mathrm{a}$.

\section{Chandrasekhar and Landau}


The name that is attached to the limiting mass of white dwarfs is that of the Indian astrophysicist Subramanian Chandrasekhar, who was also working on the structure of white dwarfs in the early 1930s. He read Stoner's paper-The limiting density of white dwarf stars [9] and realized that, with Fowler's equation of state (2), the density as a function of radius is given by the solution of the Lane-Emden equation with polytropic index $n=3 / 2$. This solution is given in tabulated form in Eddington's book The Internal Constitution of the Stars [8] so he was able to rederive Stoner's result for the dependence of density on the mass, i.e. $\rho \propto M^{2}$, without making Stoner's constant-density approximation [14]. The next year, following the publication of Stoner's second paper The Equilibrium of Dense Stars [11], Chandrasekhar applied the theory of polytropic gas spheres once again but this time with the equation of state (5) which applies only in the extreme relativistic limit. The solution of the stellar structure equations with this equation of state is a polytrope of index $n=3$. This solution is also tabulated in Eddington's book, and yields the unique mass 0.91 solar masses (1.4 $M_{\text {solar }}$ with $\mu_{e}=2$ ) which Chandrasekhar took, without proof, to be the maximum mass [15] of a white dwarf. By comparison, Stoner's maximum mass, arrived at with the constant-density approximation, was about $20 \%$ higher than this. It seems that at this stage Chandrasekhar did not understand the full significance of his result [16]. Finally four years later Chandrasekhar succeeded in solving the stellar structure equations with the StonerAnderson equation of state for the mass-radius relation for all stellar masses up to the limiting mass [17]. The solutions show that, as the limit is approached, the radius of the star shrinks to zero and the density goes to infinity. In practice the equation of state ceases to be valid when the electron energies exceed the threshold for inverse beta decay to occur, so the limiting mass is never reached.

It is interesting to note that $\mathrm{L}$ Landau also obtained a value of $M \sim 1.5 M_{\text {solar }}$ for the limiting mass of stars supported by relativistic electron degeneracy pressure [18]. But then, surprisingly, he went on to interpret this limit as arising from a failure of quantum mechanics:

'As in reality such masses exist quietly as stars and do not show any such ridiculous tendencies we must conclude that all stars heavier than $1.5 \mathrm{M}_{\text {solar }}$ certainly possess regions in which the laws of quantum mechanics (and therefore of quantum statistics) are violated'.

\section{Discussion}

As has often been the case in the history of science an important discovery is not solely due to one person. Such is the case with the discovery of a limiting mass for white dwarf stars. Stoner's paper The equilibrium of Dense Stars [11] was the first paper to give a convincing demonstration of the existence of a limiting mass. So why is it that Chandrasekhar's name is the one that has subsequently come to be associated with this discovery and why has the important role played by Stoner has been largely forgotten? There seem to be several 
reasons for this neglect of Stoner's work. Chandrasekhar pursued the study of white dwarfs further than Stoner and was the first person to carry out the accurate numerical integration of the stellar structure equations and to obtain the value 1.4 solar masses [16]. Also he tended to give rather sparing acknowledgment of Stoner's pioneering work in his papers and in the numerous interviews he gave later in his life. For a more detailed account of this matter see the article by M Nauenberg [19]. A conspicuous example of this neglect occurred In 1983 when Chandrasekhar was awarded a Nobel Prize for his work on stars. His Nobel lecture entitled On Stars and their Stability recounted in some detail the steps leading to the discovery of a limiting mass for white dwarfs. Surprisingly, in this account, acknowledgement of Stoner's work is completely absent.

Finally there is the often recounted story of the dramatic meeting of the Royal Astronomical Society that took place on $11^{\text {th }}$ January 1935 [20] in which Chandrasekhar presented his results, only for Eddington to get up and ridicule them with the words

'The star has to go on radiating and radiating and contracting and contracting until I suppose it gets down to a few kilometres radius when gravity becomes strong enough to hold in the radiation and the star can at last find peace.... I think there should be a law of nature to prevent a star behaving in this absurd way'.

Stoner's name does not appear in this famous story and he does not appear to have taken part in the subsequent debate with Eddington over the existence of a limiting mass for white dwarfs.

Such was Eddington's preeminent position as the world's leading astrophysicist that, although many of the leading physicists of the day, such as Fowler, Rosenfeld, Bohr, Dirac and Pauli were convinced that Eddington was wrong, none of them were prepared to challenge him openly [20] [21]. Most probably it was Eddington's unyielding opposition to the idea of gravitational collapse that delayed the exploration of its full implications until the 1960 s when research into black holes began.

\section{Acknowledgements}

should like to thank Edward Davis, Richard Jameson, Malcolm Longair, Peter Maksym and Derek Raine for reading the manuscript and offering helpful suggestions.

\section{References}

1. L F Bates (1969) Biographical Memoires of Fellows of the Royal Society 15 201-237.

2. E C Stoner (1924) 'The Distribution of Electrons among Atomic Levels' Philosophical Magazine 47 719-736. 
3. R H Fowler (1926) 'On Dense Matter' Monthly Notices of the Royal Astronomical Society 37 114-122.

4. M Zeilik and S A Gregory (1998) Introductory Astronomy and Astrophysics 4th Ed. Saunders Publishing.

5. F W Herschel (1785) Philosophical Transactions of the Royal Society of London 75 40-126.

6. W S Adams (1925) Proceedings of the National Academy of Sciences

7. M Barstow et al. (2005) 'HST Spectroscopy of the Balmer Lines in Sirius B' Monthly Notices of the Royal Astronomical Society 3621134.

8 A S Eddington (1926) 'The Internal constitution of the Stars' First edition Dover 1959 pp.

9. E C Stoner (1929) 'The limiting density in White Dwarf Stars' Philosophical Magazine 7,7: 41 63-70.

10. W Anderson (1929) 'Uber die Grenzdichte der Materie und der Energie' Zeitschrift fur Physik 54 851-856.

11. E C Stoner (1930) 'The Equilibrium of Dense Stars' Philosophical Magazine, 9:60,944-963.

12. D Garfinkle (2009) 'The Planck Mass and the Chandrasekhar limit' American Journal of Physics 77 683-687.

13. E C Stoner and F Tyler (1931) 'A Note on Condensed Stars', Philosophical Magazine,11:72, 986-995.

14. S Chandrasekhar (1931) 'The Density of White Dwarfs' Philosophical Magazine 11 592597.

15. S Chandrasekhar (1931) 'The Maximum Mass of Ideal White Dwarfs' Astrophysical Journal 74 81-82.

16. S Weart in an interview with Chandrasekhar in May 1977 Neils Bohr Library, American Institute of Physics.

17. S Chandrasekhar (1935) 'The Highly Collapsed Configurations of a Stellar Mass (second paper)' Monthly Notices of the Royal Astronomical Society 95 207-225.

18. L Landau (1932) 'On the Theory of Stars' Physicalische Zeitschrift der Sowjetunion 1285 288.

19. M Nauenberg 'Edmund C Stoner and the Discovery of the Maximum Mass of White Dwarfs' The Journal for the History of Astronomy August 2008.

20. A I Miller (2005) 'Empire of the Stars' (Little, Brown). 
21. M S Longair (2006) 'The Cosmic Century' (footnotes to Chapter 4), Cambridge University Press. 
This article was downloaded by: [Davis, Edward]

On: 4 January 2011

Access details: Access Details: [subscription number 908838490]

Publisher Taylor \& Francis

Informa Ltd Registered in England and Wales Registered Number: 1072954 Registered office: Mortimer House, 3741 Mortimer Street, London W1T 3JH, UK

Philosophical Magazine

\section{Philosophical Magazine Series 7}

Publication details, including instructions for authors and subscription information:

http://www.informaworld.com/smpp/title $\sim$ content=t910079897

\section{PLEASE SCROLL DOWN FOR ARTICLE}

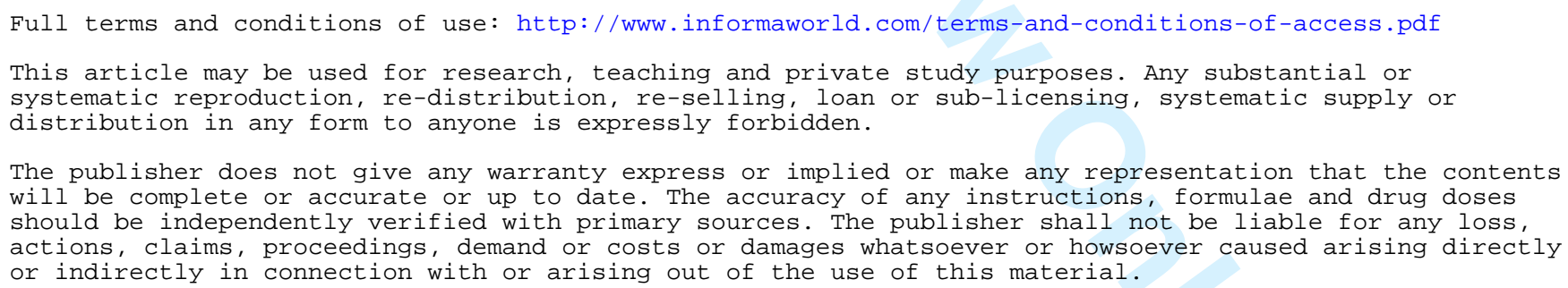


LXXXVII. The Equilibrimm of Tense Stars. By Edmund C. Stoner, Ph.D. (Camb.), Reader in Physics, University of Leeds*.

\section{Introduction.}

T $N$ white dwarf stars the last stages of the ionization 1 process are reached, so that at least in the central parts the molecules consist almost entirely of atomic nuclei and free electrons. Under these conditions very high densities are possible. The mean densities deduced from observations, though large (of the order 50 to 100 thousand), fall very far short of those corresponding to a "closepacked" arrangement, if a naive view of the "sizes" of electrons and nuclei is taken. It was suggested that some limitation might be introduced by the " jamming" of a few remaining atoms with K-ring electrons. In a previous paper $\dagger$, however, it was shown that a limitation is imposed by the exclusion principle applying to free electrons, as embodied in the Fermi statistics. The number of electrons with momenta within a definite range cannot exceed a certain maximum. Any increase in density involves an increase of energy. In the limiting case, at absolute zero, the star can contract until the decrease in gravitational energy becomes insufficient to balance the increase of kinetic energy of the electrons. With a few simplifying assumptions, an expression was derived which indicated that the maximum density varied as the square of the mass of the star. It has been pointed out by Auderson $\ddagger$ that in this calculation the effect of the relativity change of mass was neglected. He has taken this effect into account, but in a manner which seems open to criticism. His general conclusions, which seem to be correct, are that the simple expression holds provided the electron densities are not too large, but that the mass corresponding to large electron densities is smaller than that previously calculated, and that it reaches a limit. The correction becomes important for stars of mass about half that of the Sun, and so for the white dwarfs which were actually considered. The main purpose of this paper is to calculate the effect of the relativity change of mass, using a method which seems more rigorous than

* Communicated by Prof. R. Whiddington, F.R.S.

+ E. C. Stoner, Phil. Mag. vii. p. 63 (1929). (This paper will be referred to as I.)

† W. Anderson, Zeits. fiir Phys. lvi. p. 851 (1929). 
that of Anderson. The conclusion drawn is that the range in which the simple expression holds approximately is wider than that indicated by Anderson's results, the "limiting mass" being somewhat greater, so that there is still an equilibrium state, under the particular conditions, for the stars under consideration. The significance of the results for very dense stars is discussed, and the effect of the electron gas following the Fermi statistics at the smaller densities found in normal stars is briefly considered.

\section{Efject of the Relativity Change of Mass on the Zero-point Energy of Electron Gas.}

Let $\mathrm{E}_{\mathrm{G}}$ be the gravitational energy of the idealized star, $E_{K}$ the total kinetic energy of the electrons, $n$ the number of electrons per unit volume. Then, as previously shown, the equilibrium condition is given by

$$
\frac{d}{d n}\left(\mathrm{E}_{\mathrm{G}}+\mathrm{E}_{\mathrm{K}}\right)=0 . . \quad . \quad . \quad .
$$

Let $\bar{\epsilon}$ be the mean kinetic energy of the electrons at absolute zero, $V$ the volume of the star, supposed to be of uniform density. (The distribution of density will be considered later.) When the relativity change of mass is neglected, the total kinetic energy at absolute zero is given by

$$
\mathrm{E}_{\mathrm{K}}=n \mathrm{~V} \bar{\epsilon}=n \mathrm{~V} \frac{3}{40}\left(\frac{3}{\pi}\right)^{2 / 3} \frac{h^{2} n^{2 / 3}}{m_{0}} . \quad . \quad .
$$

It is this expression which has to be modified.

In Anderson's treatuent $m_{0}$ is replaced by $m$, where $m$ is derived from the equation

$$
c^{2}\left(m-m_{0}\right)=\frac{3}{40}\left(\frac{3}{\pi}\right)^{2 / 3} \frac{h^{2} n^{2 \cdot 3}}{m} . . . .
$$

It is thus assumed that the total kinetic energy can be expressed in the alternative forms

$$
n V c^{2}\left(m-m_{0}\right) \quad \text { and } \quad n V \frac{3}{40}\left(\frac{3}{\pi}\right)^{2 / 3} \frac{h^{2} n^{3 / 3}}{m},
$$

where $m$ has the same value in both expressions, being a "mean" mass ; further, that this mean mass can be employed in subsequent calculations in place of $m_{0}$. The value of it mean mass, however, depends on the manner in which the mass enters into the particular expressions for which the appropriate mean value is being derived, and it is not legitimate to use one particular meau value indiscriminately

$$
\text { Phil. Mag. S. 7. Vol. 9. No.60.May 1930. } 3 \mathrm{Q}
$$


in more general calculations. (Perhaps the simplest illustration is that the arithmetic mean is not in general equal to the root mean square. The objection to Anderson's treatment has a similar basis.) It seems more satisfactory to attack the problem of determining the zero-point energy without introducing the idea of mean mass at all.

Let $\epsilon$ be the kinetic energy of an electron, $p$ the momentum, and let $\beta=v / c$,

$$
\begin{gathered}
\epsilon=\left(m-m_{0}\right) c^{2}=m_{0} c^{2}\left(\frac{1}{\sqrt{1-\beta^{2}}}-1\right), \\
p^{2}=(m \beta c)^{2}=m_{0}^{2} c^{2}\left(\frac{1}{1-\beta^{2}}-1\right) . . .
\end{gathered}
$$

Substituting for $1 / \sqrt{1-\beta^{2}}$ in (4),

$$
\epsilon=m_{0} c^{2}\left[\left(1+\frac{p^{2}}{m_{0}^{2} c^{2}}\right)^{1 / 2}-1\right] \ldots . . .
$$

In the completely condensed state there are two electrons in each cell of the 6-dimensional phase-space (of 6 -volume $h^{3}$ ), so that the number of electrons in a volume $V$ with momenta between $p$ and $p+d p$ is equal to

$$
2 \times \frac{4 \pi p^{2} d p}{h^{3}} \times \mathrm{V}
$$

Thus for the total energy $\mathrm{E}_{\mathrm{K}}$ of the electrons in a condensed state with maximum momentum $p_{0}$

$$
\mathrm{E}_{\mathrm{K}}=\int_{0}^{p_{0}} 2 m_{0} c^{2}\left[\left(1+\frac{p^{2}}{m_{0}^{2} c^{2}}\right)^{1 / 2}-1\right] \frac{4 \pi p^{2} d p V}{h^{3}} .
$$

Since $n V$ is the total number of electrons (double the number of cells), the maximum momentum $p_{0}$ is related to $n$ by

$$
\begin{aligned}
& \left(\mathrm{V} / h^{3}\right) \int_{0}^{p_{0}} 4 \pi p^{2} d p=\frac{1}{2} n \mathrm{~V} . \\
& \left.n=\frac{8 \pi p_{0}^{3}}{h^{3}}, \quad p_{0}=\left(\frac{3 h^{3} n}{8 \pi}\right)^{1 / 3} \cdot\right)
\end{aligned}
$$

Let 
Then

$$
\begin{aligned}
\mathrm{E}_{\mathrm{K}} & =\frac{8 \pi \mathrm{V} m_{0} c^{2}}{h^{3}} \times\left(m_{0} c\right)^{3} \int_{0}^{x}\left\{\left(1+y^{2}\right)^{1 / 2}-1\right\} y^{2} d y, \\
& =\frac{8 \pi \mathrm{V} m_{0} c^{5}}{h^{3}}\left[-\frac{x^{3}}{3}+\int_{0}^{x}\left(1+y^{2}\right)^{1 / 2} y^{2} d y\right] . \\
& =\frac{8 \pi \mathrm{V} m_{0}^{4} c^{5}}{h^{3}}\left[-\frac{x^{3}}{3}+f(x)\right] . . . . . . .
\end{aligned}
$$

As long as $x$ is small (i. e., $p_{0}<<m_{0} c$ ), $f(x)$ may be readily evaluated by integration in series,

$$
\begin{aligned}
& {[f(x)]_{x<}=\frac{1}{3} x^{3}+\frac{1}{10} x^{5}-\frac{1}{56} x^{7} \ldots,} \\
& \mathrm{E}_{\mathrm{K}}=\frac{8 \pi \mathrm{V} m_{0}{ }^{4} c^{5}}{10 h^{3}} x^{5}\left(1-\frac{5}{28} x^{2} \ldots\right) ., .
\end{aligned}
$$

Substituting for $x$

$$
\begin{aligned}
\mathrm{E}_{\mathrm{K}} & =\frac{8 \pi \mathrm{V}}{3 h^{3} m_{0}} p_{0}{ }^{5}\left(1-\frac{5}{28} \frac{p_{0}{ }^{2}}{m_{0}{ }^{2} c^{2}} \ldots\right), \quad . \quad . . \\
& =n \mathrm{~V} \frac{3}{40}\left(\frac{3}{\pi}\right)^{2 / 3} \frac{h^{2} n^{2 / 3}}{m_{0}}\left[1-\frac{5 h^{2}}{112}\left(\frac{3}{\pi}\right)^{2 / 3} n^{2 / 3}\right], .
\end{aligned}
$$

agreeing in the limit with (2) for $n$ small.

The above expression may be used for $p_{0} \ll m_{0} c$, corresponding to $n<\frac{8 \pi}{3}\left(\frac{m_{0} c}{h}\right)^{3}$, or, substituting numerical values, $n<<5.88 \times 10^{29}$. For stars of mass 44 and $\cdot 85$ that of the Sun, the value of $n$ came out on the simple theory as about $1.86 \times 10^{29}$ and $6.9 \times 10^{29}$. The approximation given by series integration is thus inadequate, and it is necessary to use the complete expression for the integral

$$
\begin{aligned}
f(x) & =\int_{0}^{x}\left(1+y^{2}\right)^{1 / 2} y^{2} d y \\
& =\int_{0}^{x}\left(1+y^{2}\right)^{3 / 2} d y-\int_{0}^{x}\left(1+y^{2}\right)^{1 / 2} d y .
\end{aligned}
$$

The second integral is known, and the first may be reduced by noting that

$$
\begin{aligned}
\frac{d}{d y}\left\{y\left(1+y^{2}\right)^{1 / 2}\right\}= & 4\left(1+y^{2}\right)^{3 / 2}-3\left(1+y^{2}\right)^{1 / 2} . \\
& 3 Q 2
\end{aligned}
$$

http://mc.manuscriptcentral.com/pm-pml 
Page 15 of Bailosophical Magazine \& Philosophical Magazine Letters

948

\author{
Dr. E. C. Stoner on the
}

The final result is

$$
f(x)=\frac{1}{8}\left[x\left(1+x^{2}\right)^{1 / 2}\left(1+2 x^{2}\right)-\log \left\{x+\left(1+x^{2}\right)^{1 / 2}\right\}\right] .
$$

For $x$ small this may be expanded (noting that the $\log$ is equal to

$$
\left.\int\left(1+x^{2}\right)^{-1 / 2} d x\right)
$$

giving the result (10) on substitution in $(9 a)$.

The complete expression for the total kinetic energy of the electrons thus becomes

$$
\mathrm{E}_{\mathrm{K}}=\frac{8 \pi \mathrm{V} m_{0}{ }^{4} c^{5}}{h^{3}}\left[\frac{1}{8} x\left(1+x^{2}\right)^{1 / 2}\left(1+2 x^{2}\right)-\frac{1}{3} x^{3}\right.
$$

with

$$
\left.-\log \left\{x+\left(1+x^{2}\right)^{1 / 2}\right\}\right] \text {. }
$$

$$
x=\frac{p_{0}}{m_{0} c}=\frac{1}{m_{0} c}\left(\frac{3 h^{3} n}{8 \pi}\right)^{1 / 3} .
$$

The energy is less than that given by (2). In the limit when $x$ is large $\left(x>>1, n>>5.88 \times 10^{29}\right)$,

$$
\begin{aligned}
\left(\mathrm{E}_{\mathrm{K}}\right)_{x>>1} & =\frac{8 \pi \mathrm{V} m_{0}{ }^{4} c^{5}}{\ldots h^{3}} \frac{x^{4}}{4} \\
& =\frac{2 \pi \mathrm{V} c}{h^{3}} p_{0}{ }^{4}=n \mathrm{~V} \frac{3}{8}\left(\frac{3}{\pi}\right)^{1 / 3} h c n^{1 / 3} .
\end{aligned}
$$

These expressions may be compared with (10a) and (10b). They show that when $n$ is large the mean kinetic energy increases as $n^{1 / 3}$ (instead of $n^{2}$ ). For a constant total number of electrons ( $n \mathrm{~V}$ constant), $\mathrm{E}_{k}$ then varies as $n^{1 / 3}$, as does also $-E_{G}$. When this is so there will obviously be no equilibrium under the conditions imposed.

\title{
The Equilibrium Condition.
}

If the conditions are such that an equilibrium state is possible, there will be a limiting density-_limiting" because the calculation refers to a sphere of uniform density at zero temperature. The limiting density is that corresponding to the value of $n$ when

$$
\frac{d}{d n}\left(\mathbf{E}_{\mathrm{x}}+\mathbf{E}_{G}\right)=0 \text {. . . . . . }
$$

It is more convenient, for the present application, to treat $x$, defined by (8), as the variable, so that the equilibrium condition becomes

$$
\frac{d}{d x}\left(\mathrm{E}_{\mathrm{K}}+\mathrm{E}_{\mathrm{G}}\right)=0 . \quad \text {. . . . . . }
$$


For the gravitational potential energy (as shown in I.) with $2.5 m_{\mathrm{H}}$ as the mean molecular weight of the material of the star,

$$
\mathrm{E}_{\mathrm{G}}=-\frac{3}{5} \frac{\mathrm{GM}^{2}}{\mathrm{M}^{1 / 3}}\left(\begin{array}{l}
4 \\
3
\end{array}{ }^{n n}\right)^{1 / 3}\left(2 \cdot 5 m_{\mathrm{H}}\right)^{1 / 3} \cdot .
$$

Substituting $\left(\frac{8 \pi}{3}\right)^{1 / 3} \frac{m_{0} c x}{h}$ for $n^{1 / 3}$ from (8),

$$
\mathrm{E}_{\mathrm{G}}=3^{1 / 3}\left(\frac{4 \pi}{5}\right)^{2 / 3} \frac{1}{h} \mathrm{GM}^{5 / 3} m_{\mathrm{H}}^{1 / 3} m_{0} c x . \quad .
$$

From (12) $\mathrm{E}_{\mathrm{k}}$ may be written as

$$
\mathrm{E}_{\mathrm{K}}=\frac{8 \pi \mathrm{V} m_{0}{ }^{4} c^{5}}{h^{3}} f_{1}(x)
$$

Substituting $\mathrm{M} /\left(2 \cdot 5 m_{\mathrm{H}} n\right)$ for $\mathrm{V}$, and further substituting for $n$ as above,

$$
\mathrm{E}_{\mathrm{K}}=\frac{3 \mathrm{M} m_{0} c^{2}}{2 \cdot 5 m_{\mathrm{H}}} \frac{f_{1}(x)}{x^{3}} \cdot \ldots \cdot \cdot \cdot .
$$

Jsing the values (15) and (16) in (14), the result is

$$
\frac{d}{d x}\left[\frac{1}{x^{3}} f_{1}(x)\right]=10^{1 / 3}\left(\begin{array}{l}
\pi \\
3
\end{array}\right)^{2 / 3} \frac{\mathrm{Gm}_{\mathrm{H}}{ }^{4 / 3} \mathrm{M}^{2 / 3}}{h c} .
$$

Inserting numerical values,

$$
\begin{gathered}
\frac{d}{d x}\left[\frac{1}{x^{3}} f_{1}(x)\right]=\mathrm{F}(x)=1.483 \times 10^{-23} \mathrm{M}^{2 / 3}, . \\
\mathrm{M}=1.751 \times 10^{34}[\mathrm{~F}(x)]^{3 / 2} \ldots . . .
\end{gathered}
$$

$\mathbf{F}(x)$ is obtained by straightforward differentiation of $\frac{1}{x^{3}} f_{1}(x)$, where $f_{1}(x)$ is the bracketed quantity in (12). The final result is

$$
\mathrm{F}(x)=\frac{1}{8 x^{3}}\left[\frac{3}{x} \log \left\{x+\left(1+x^{2}\right)^{1 / 2}\right\}+\left(1+x^{2}\right)^{1 / 2}\left(2 x^{2}-3\right)\right] .
$$

By means of equations (18a) and (19) the mass may be found corresponding to any value of $x$, and so to any limiting electron concentration. Since the mean molecular weight is about $2 \cdot 5 \mathrm{~m}_{\mathrm{H}}$, the limiting density is given by

$$
\rho_{0}=2 \cdot 5 m_{\mathrm{H}} n=4 \cdot 15 \times 10^{-24} n \text {. . . }
$$




\section{Numerical Results.}

The method of procedure adopted is to calculate $\mathbf{F}(x)$ for different values of $x$, from which a $\mathrm{F}(x), x$ curve may be plotted. From this, using (18 a), an $\mathrm{M}, x$ curve may be derived. If desired, this may be converted into an $M, n$ curve, using the relation

$$
n=\left(\frac{8 \pi}{3}\right)\left(\frac{m_{0} c}{h}\right)^{3} x^{3}
$$

giving

$$
n=5.876 \times 10^{29} x^{3} \quad \text { or } \quad x=1.194 \times 10^{-10} n^{1 / 3} .
$$

The full expression for $F(x)(19)$ is inconvenient to use when $x$ is small. The expression may then be expanded, with the result

$$
[\mathrm{F}(x)]_{x<1}=\frac{1}{5} x-\frac{1}{14} x^{3}+\frac{1}{24} x^{5} \ldots . .
$$

When $x$ is small enough for the first term in the expansion to be sufficient, the same result is obtained as before, namely,

$$
n=10^{4}\left(\frac{\pi}{3}\right)^{3} \frac{1}{h^{6}} \mathrm{G}^{3} \mathrm{M}^{2} m_{\mathrm{H}}{ }^{4} m^{3}, \quad \text {. . . . }
$$

or, substituting numerical values from (18a), (20), and (21),

$$
\left.\begin{array}{l}
n=2.396 \times 10^{-3 i} \mathrm{M}^{2}, \\
\rho=9.95 \times 10^{-61} \mathrm{M}^{2}
\end{array}\right\} \cdot . \quad \cdot(22)^{*}
$$

The first two terms in $(19 a)$ give $\mathrm{F}(x)$ correct to less than 1 per cent. up to $x=\cdot 5$, and form the most convenient expression for this range.

When $x$ is large, as may be seen from (19),

$$
\begin{aligned}
{[\mathrm{F}(x)]_{x>>1} } & =\frac{1}{8 x^{3}}\left(2 x^{3}-3 x\right), \\
& =\cdot 2500-\frac{3}{8 x^{2}} . . . . .
\end{aligned}
$$

This expression gives $F(x)$ correct to 1 per cent. for $x>10$. It indicates, moreover, the limiting mass for which an

* This is in agreement with Anderson's result. In I., as pointed out by Anderson, a slight numerical error was made, and the result was given as $n=2.3 \mathrm{I} \times 10^{-3^{3}} \mathrm{M}^{2}$. Correspondingly, the result for the maximum density, given as $\rho=3.85 \times 10^{6}\left(\mathrm{M} / \mathrm{M}_{s}\right)^{2}$, should be $\rho=3.977$ $\times 10^{6}\left(\mathrm{M} / \mathrm{M}_{s}\right)^{2}$, with the Sun's mass, $\mathrm{M}_{s}$, taken as $2.0 \times 10^{33}$. 
equilibrium state can be attained under the conditions, namely, from (18 $a)$,

$$
\begin{aligned}
\mathrm{M}_{0} & =1.751 \times 10^{34}(\cdot 25)^{3 / 2}, \\
& =2 \cdot 19 \times 10^{33} .
\end{aligned}
$$

\section{TABLE I.}

Corresponding values of $x, \mathrm{~F}(x), \log _{10} n$, and $\log _{10} \mathrm{M}$.

$$
\begin{aligned}
n & =5 \cdot 876 \times 10^{29} x^{3}, \\
\mathrm{M} & =1 \cdot 751 \times 10^{34}[\mathrm{~F}(x)]^{3 / 2} .
\end{aligned}
$$

\begin{tabular}{|c|c|c|c|}
\hline$x$ & $\mathbf{F}(x)$ & $\log _{10} n$ & $\log _{\delta 1 \mathrm{iv}} \mathrm{MI}$ \\
\hline$\cdot 1$ & 0200 & $26 \cdot 7690$ & 31.6947 \\
\hline$\cdot 2$ & $\cdot 039 \pm$ & $27 \cdot 6720$ & $32 \cdot 1364$ \\
\hline 3 & .0581 & $28 \cdot 2003$ & $\cdot 3895$ \\
\hline 4 & .0756 & $\cdot 5753$ & $\cdot 5609$ \\
\hline 6 & $\cdot 1071$ & $29 \cdot 1036$ & .7879 \\
\hline 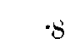 & $\cdot 1330$ & $\cdot 4783$ & .9291 \\
\hline $1 \cdot 0$ & $\cdot 1537$ & 7690 & $33 \cdot 0233$ \\
\hline 1.5 & $\cdot 1887$ & $30 \cdot 2973$ & $\cdot 1569$ \\
\hline 2 & 2085 & $\cdot 67: 0$ & $22: 20$ \\
\hline 3 & 2.200 & $31 \cdot 2003$ & $\simeq 801$ \\
\hline 4 & .2366 & $\cdot 5753$ & .3042 \\
\hline 5 & 2410 & .8660 & 3162 \\
\hline 6 & .2436 & $32 \cdot 1036$ & 3233 \\
\hline 8 & $\cdot 2463$ & $\cdot 4783$ & $\cdot 3303$ \\
\hline 10 & .2476 & 7690 & .3339 \\
\hline 20 & .2494 & $33 \cdot 6720$ & $\cdot 3385$ \\
\hline 40 & $\cdot 2498$ & $34: 0753$ & $\cdot 3396$ \\
\hline 60 & 2499 & $35 \cdot 1036$ & 3399 \\
\hline 100 & .2500 & 7690 & $3 \div 01$ \\
\hline
\end{tabular}

The value obtained by Anderson for $\mathrm{M}_{0}$ is $1.37 \times 10^{33}$, so that the range of mass in which this particular type of equilibrium can occur is shown by this method to be considerably greater than that indicated by Anderson's approximate treatment.

A series of corresponding values of $x$ and $\mathrm{F}(x)$ calculated from (19), supplemented by $(19 a)$ and $(19 b)$, is shown in Table I. The relation required is that between $n$ and M, related to $x$ and $\mathrm{F}(x)$ by $(21)$ and $(18 a)$. It is convenient 
Page 19 of Bailosophical Magazine \& Philosophical Magazine Letters

952

Dr. E. C. Stoner on the

to use logarithms (base 10) when the following equations are obtained :

$$
\begin{aligned}
& \log _{10} n=29 \cdot 7690+3 \log x, \quad . \quad . \quad . \quad . \\
& \log _{10} \mathrm{M}=34 \cdot 2432+\frac{3}{2} \log \mathrm{F}(x) . \quad . \quad . \quad .
\end{aligned}
$$

The conversion is then readily carried out. Corresponding values of $n$ and $\mathrm{M}$ are shown in the last two columns of the table. It is unnecessary to give values for $\log M$ less than 32, as no stars are known of mass less than a tonth that of the $\operatorname{Sun}\left(\log \mathrm{M}_{s}=33.3010\right)$, and in any case, for stars of small mass, the approximate expression (22) will be sufficiently accurate. For any value of $M$ the maximum value of $\log n$ can be fornd by interpolation, and from that

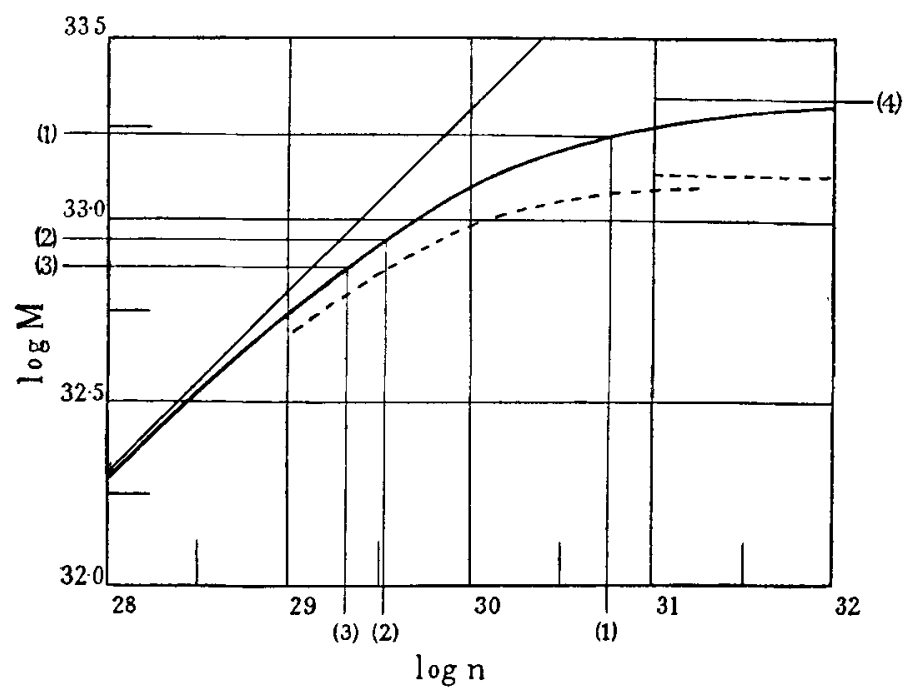

Variation of limiting electron concentrution $(n)$ with mass (M) in a sphere of uniform deusity.

(1) Sirus $\mathrm{B} \quad \ldots \ldots, \log \mathrm{M}=33 \cdot 230 \quad \log n=30 \cdot 748$

(2) $o_{2}$ Eridani $B \ldots \log M=32 \cdot 944 \quad \log n=29 \cdot 524$

(3) Procyon B ..... $\log \mathrm{M}=32 \cdot 869 \quad \log n=29 \cdot 313$

(4) Limiting $M \ldots . \log M_{n}=33 \cdot 340 \quad\left(M_{0}=2 \cdot 19 \times 10^{23}\right)$

For the limiting density $\log \rho_{0}=\log n-24+0 \cdot 618$.

The straight line corresponds to the formula in which the relativity effect is neglected. The dotted curve gives Anderson's results.

the value of $n$. If the density is required it can be found from (20), while $\log \rho_{0}$ is given by

$$
\log \rho_{0}=\log n-24+\cdot 6180 \text {. . . . . }
$$


The relation betwe $\mathbf{n} \log M$ and $\log n$ is shown by the curve in the figure. "The "positions" of a number of stars are indicated. The straight line is the result found by the original approximation, in which the relativity effect was neglected (giving $n$ varying as $\mathrm{M}^{2}$ ). The main conclusion is that for stars for which this equilibrium can occur the limiting density is somewhat less than that calculated by Anderson, and that the "limiting mass" is larger; his results are indicated by the dotted line.

\section{White Dirarfs.}

The number of stars known to be of the white dwarf type is small, but this does not necessarily indicate that stars of very high density are uncommon. Dense stars of ordinary mass will have a small radius, and so will be faint objects unless they are near the Sun (on the stellar scale of nearness) or have a high-surface temperature. "Black dwarfs" (to use Fowler's term) would not be observed. According to Jeans *, four white dwarfs are known with certainty, but for one of these-the companion of the red giant o Ceti-he does not give any quantitative data. Procyon $B$ is a possible white dwarf, but its spectral type is unknown. In the following table are collected the relevant observational data derived from Jeans $\dagger$. Procyon $B$ is included because its mass is known. The four columns give the spectral type, the absolute visual and bolometric magnitudes, and the mass in terms of the Sun's mass $\left(\mathrm{M}_{s}\right)$.

\section{TABLE II.}

Observational Data.

\begin{tabular}{|c|c|c|c|c|}
\hline & $\begin{array}{l}\text { Spectral } \\
\text { Type. }\end{array}$ & $\mathbf{M}_{\text {ris. }}$ & $\mathbf{M}_{\text {bol. }}$ & $\mathrm{M} / \mathrm{M}_{*}$ \\
\hline simus $B \quad \ldots \ldots \ldots \ldots \ldots$ & \pm 7 & $11 \cdot 3$ & $11 \div 2$ & $\cdot 85$ \\
\hline$o_{z}$ Eridani B $\ldots \ldots \ldots \ldots$ & AO & $11 \cdot 2$ & $10 \cdot 8$ & $\cdot 44$ \\
\hline Proevon $B \quad \ldots \quad \ldots \ldots \ldots$. & - & 16 & $\cdots$ & $\cdot 37$ \\
\hline Van Maanen's star ...... & $\mathrm{F}$ & $1+3$ & $14 \cdot 3$ & - \\
\hline
\end{tabular}
1928 ).

* J. H. Jeans, 'Astronomy and Cosmogony,' p. 63 (Camb. Univ. Press, + Loc. cit. p. 59. I am indebted to Prof. Eddington for informing me that the value 21 given for the mass of $o_{2}$ Eridani $B$ in his 'Internul Constitution of the Stars' is based on an earlier determination of the orbit, and that the value $\cdot 44$ is presumably the more trustworthy. Also to Sir James Jeans for confirming the value $\cdot \mathbf{4 4}$. 
From these observational data deductions may be made as to other characteristics of the stars. From the spectral type the surface temperature $\left(\mathrm{T}_{e}\right)$ may be estimated, and from the effective temperature and the absolute magnitudes the radius of the star. The following formulæ are given by Jeans for the radius in terms of that of the sun $\left(r / r_{s}\right.$ denoted by $\left.\mathrm{R}\right):-$

$$
\begin{aligned}
& \log \mathrm{R}=-0.2 \mathrm{M}_{\text {bol. }}-2 \log \mathrm{T}_{e}+8.53, \ldots \\
& \log \mathrm{R}=-0.2 \mathrm{M}_{\text {ris. }}+\frac{5880}{\mathrm{I}_{e}}-0.01 . .
\end{aligned}
$$

It is further possible to calculate the total radiation of energy in ergs per second (E),

$$
\log \mathrm{E}=-0.4 \mathrm{M}_{\text {bol. }}+35.52 \text {. . . . }
$$

\begin{tabular}{|c|c|c|c|c|c|c|}
\hline & $\mathrm{M} / \mathrm{M}_{\mathrm{s}}$ & $\mathrm{I}^{\prime}$ & R. & $\mathrm{E}^{\prime}$ & $\rho$. & $\rho_{0}$ calc. \\
\hline Sirius B & .85 & 8,000 & .0312 & $\cdot 0065$ & $3.97 \times 10^{4}$ & $2.3 \times 10^{7}$ \\
\hline$o_{2}$ Eridani B... & $\cdot 44$ & 11,200 & $\cdot 0187$ & 0185 & $9.55 \times 10^{4}$ & $1.4 \times 10^{6}$ \\
\hline
\end{tabular}

TABLe III.

Deduced Characteristics of Sirius B and $o_{2}$ Eridani B.

The amount of radiation per gram $\left(E^{\prime}\right)$ is a quantity of great interest:

$$
\begin{aligned}
\log \mathrm{E}^{\prime} & =\log \mathrm{E}-\log \mathrm{M}, \\
& =\log \mathrm{E}+\log \left(\mathrm{M}_{s} / \mathrm{M}\right)-\log \mathrm{M}_{s}, \\
& =-0 \cdot 4 \mathrm{M}_{\mathrm{bol.}}+2 \cdot 22+\log \left(\mathrm{M}_{s} / \mathrm{M}\right) .
\end{aligned}
$$

(The sun's mass is taken as $2 \cdot 0 \times 10^{33}$ grams.)

The mean density is given by

$$
\begin{aligned}
\rho & =\frac{3 \mathrm{M}}{4 \pi r^{3}}=\left(\frac{\mathrm{M}}{\mathrm{M}_{s}}\right)\left(\frac{r_{s}}{r}\right)^{3} \frac{3 \mathrm{M}_{s}}{4 \pi r_{s}{ }^{3}}, \\
& =\left(\mathrm{M} / \mathrm{M}_{s}\right)(1 / \mathrm{R})^{3} \rho_{s}=1.42\left(\mathrm{M} / \mathrm{M}_{s}\right)(1 / \mathrm{R})^{3} . . .
\end{aligned}
$$

The values given in Table III. have been calculated from these equations. It may be noted that for Sirius $B$ the radius deduced from the relativity shift is $\cdot 03$, in remarkable agreement with the value 0312 calculated in the way indicated. (In general the values are not significant to more than two figures.) The available data for Procyon B and Van Maanen's Star are insufficient for the calculation 
of $\rho$ or $\mathrm{E}^{\prime}$; these stars will be discussed later. The value of $M / M_{s}$ is included in the table, and also the value of the limiting density, $\rho_{0}$, calculated as already described.

The calculated limiting densities are considerably greater than those obtained by the simple formula (19a) (used in I.), which gives for stars of the mass of Sirius $B$ and $o_{2}$ Eridani $B$ the values $\rho=2.8 \times 10^{6}$ and $7.5 \times 10^{5}$. The order of magnitude of the difference for stars of different mass will be apparent from the figure.

The calculated radiation per gram $\left(\mathrm{E}^{\prime}\right)$ of $\mathrm{O}_{2}$ Eridani B is about 10 times greater than the value given by Jeans. This will be discussed later. The mean density for Sirius B is somewhat smaller than that usually given (about $5 \times 10^{4}$ ). This results from the use of a slightly larger value for the radius. Taking the relativity shift value $(K=.03)$, the mean density comes out as $4.5 \times 10^{5}$.

\section{Mean Molecular Weight.}

The mean molecular weight has been taken as $2.5 m_{\mathrm{H}}$. This is the value for completely ionized atoms of lead $\left(\frac{207 \cdot 2}{82+1}\right)$. For atoms of lower atomic number the value is slightly smaller, and for those of high atomic number, larger. Jeans derives a value $2.6 m_{\mathrm{H}}$ on the assumption, for which there is strong evidence, that in many stars atoms of the atomic number equal to that of uranium, and even higher, are present. In condensed stars of the type considered, however, most of these atoms will have been transformed into atoms of an inert type, and the estimate $2.5 m_{\text {H }}$ is probably sufficiently accurate. In any case, a small change will not affect the numerical results appreciably.

It might be thought that the change of mass of the electron would produce a not negligible effect, but it may readily be shown that the effect will be very small. Let $m$ be the mass of an electron with momentum $p$. The molecular weight becomes $2 \cdot 5 m_{\mathrm{H}}+\mu$, where

Now

$$
2 \cdot 5 m_{\mathrm{H}}+\mu=m_{\mathrm{H}}\left(2 \cdot 5+\frac{m-m_{0}}{m_{\mathrm{Hr}}}\right) .
$$

$$
\begin{aligned}
m^{2}(1-\beta) & =m_{0}^{2}, \\
\beta & =\frac{p}{m c}=\left(-\frac{p}{m_{0} c}\right) \frac{m_{0}}{m}
\end{aligned}
$$




$$
\begin{gathered}
\text { Dr. E. C. Stoner on the } \\
\frac{m-m_{0}}{m_{0}}=\left[1+\left(\frac{p}{m_{0} c}\right)^{2}\right]^{1 / 2}, \\
2 \cdot 5 m_{\mathrm{H}}+\mu=m_{\mathrm{H}}\left[2 \cdot 5+\frac{1}{1845}\left\{1+\left(\frac{p}{m_{0} c}\right)^{2}\right\}^{1 / 2}\right] .
\end{gathered}
$$

From (8) the maximum momentum is given by

so that

$$
p_{0}=\left(\frac{3 h^{3} n}{8 \pi}\right)^{1 / 3}
$$

$$
\begin{aligned}
\frac{p_{0}}{m_{0} \mathrm{c}} & =\frac{h}{m_{0} c}\left(\frac{3}{\zeta \pi}\right)^{1 / 3} n^{1 / 3}, \\
& =1.194 \times 10^{-10} n^{1 / 3} . \quad . \quad . .
\end{aligned}
$$

For an electron concentration $n=10^{33}$ (corresponding to a density greater than $10^{9}$ ) the maximum molecular weight is

$$
2 \cdot 5 m_{\mathrm{H}}+\mu=m_{\mathrm{s}}(2 \cdot 5+\cdot 0065) \text {. }
$$

The correction is thus quite negligible for all the densities which come into consideration.

\section{Mean Density.}

In the foregoing treatment the star has been idealized as having a uniform density. This seems legitimate when the aim has been to obtain an estimate of the maximum density under gravitational-kinetic equilibrjum. In an aetual star, however, the density will not be uniform, even in the condensed limit. When $n$ is small the energy per unit volume is proportional to $x^{5 / 3}$ (eq. $10 b$ ), and when $n$ is large, to $n^{4 / 3}$ (eq. 12a). Since the pressure is proportional to the energy per unit volume, and the density is proportional to $n$, the following relations hold between the pressure and density :-

$$
\begin{array}{ll}
n<<5.9 \times 10^{29}, & \rho<2.4 \times 10^{6}, \quad p=\kappa \rho^{5 / 3}, . \\
n \gg 5.9 \times 10^{29}, & \rho^{\prime}>>2.4 \times 10^{6}, \quad p=\kappa \rho^{4 / 3} .
\end{array}
$$

In an ideal condensed star the distribution will thus he polytropic, the relation between pressure and density obeying the relation $p=\kappa \rho^{\gamma}$. The distributions in polytropie stars for a number of values of $\gamma$ (or its equivalent $n^{\prime}$, defined by $\left.\gamma=1+1 / n^{\prime}\right)$ have been worked out by Emden. 'The results are quoted by Eddington*. Table IV. gives the ratio of the

* A. S. Eddington, 'The Internal Constitution of the Stars,' p. 89 (C. U.P. 1926). 
maximum (central) to the mean density. Uniform density corresponds to $n^{\prime}=0$.

This table certainly indicates that the maximum mean density may be considerably less than the maximum central density, and it might seem that all that is necessary to convert the previously calculated results for limiting densities to mean densities is to divide by the appropriate ratio. There are a number of other factors, however, which have to be taken into consideration. The gravitational energy of a star is given by *

$$
\mathrm{E}_{\mathrm{G}}=-\frac{3}{5-n^{\prime}} \mathrm{G} \frac{\mathrm{M}^{2}}{r} . .
$$

For uniform density the coefficient is $3 / 5$, the value used (eq. $15 a$ ). For $\gamma=5 / 3$ the factor becomes $6 / 7$, so that for a given $\mathrm{M}$ the calculated $n$ would be increased. The whole

'Table IV.

Ratio of Maximum to Mean Density in Polytropes.

\begin{tabular}{|c|c|c|c|c|c|}
\hline$\gamma \quad \ldots \ldots \ldots . . . .$. & $\infty$ & $5 / 3$ & $3 / 2$ & $7 / 5$ & $4 / 3$ \\
\hline$n^{\prime} \ldots \ldots \ldots \ldots$ & 0 & $3 / 2$ & & $5 / 2$ & 3 \\
\hline$\rho_{0} / \rho \quad \ldots \ldots \ldots$ & 1 & 6.00 & 11.4 & $24 \cdot 1$ & $54 \cdot 4$ \\
\hline
\end{tabular}

calculation, however, is based on the assumption that $n$ is uniform, and different averaging processes would have to be carried out in deriving expressions for the gravitational and kinetic energies in terms either of $n_{0}$ (the central electron concentration) or the mean $n$. Some rough calculations indicate that the values derived for the limiting concentrations approximate to those for $n_{0}$; but i much more elaborate investigation would be necessary if the variation of density were to be properly taken into account. The indications are that the values obtained for the limiting density approach those for the limiting central density, and that the limiting mass may be slightly changed; that. Sirius B still falls within the range for which gravitationalkinetic equilibrium can occur; and that the limiting mean densitios may be smaller than those calculated approximately in the ratios suggested by the values in Table IV. These results, however, are somewhat uncertain, and for the present the calculations are left in the form which is strictly appropriate for the case of the homogeneous sphere.

* Eddington, loc. cit. p. 87 . 
Van Maanen's Star and Prosyon B.

Van Maanen's Star is of great interest, as it is the smallest star known, being about the same size as the earth. From the data in Table II. the radius may be calculated (eq. 27), the result being $R=\cdot 00955$, taking the surface temperature as 7:00 corresponding to an F-type star. The mass is unknown, and since the star is not a binary component, there is no method available by which it may be estimated. It is, however, possible to calculate compatible values of the mass (giving the mean density) and of the radiation per gram $\left(\mathrm{E}^{\prime}\right)$ from the known data. From (29),

$$
\begin{aligned}
\log \mathrm{E}^{\prime} & =-5 \cdot 72+2 \cdot 22+\log \left(\mathrm{M}_{s} / \mathrm{M}\right), \\
\mathrm{E}^{\prime}\left(\mathrm{M} / \mathrm{M}_{s}\right) & =3 \cdot 162 \times 10^{-4} .
\end{aligned}
$$

\section{TABLE V.}

Compatible Values for Van Maanen's Star.

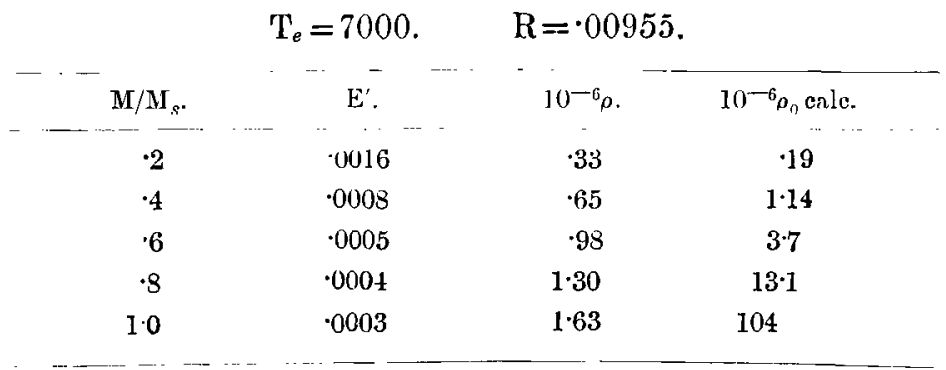

In Table $V$. are given compatible values of $M / M_{s}, \rho$, and $E^{\prime}$, together with the calculated $\rho_{0}$ for arch value of the mass. Incidentally, this table gives the values of $\rho_{0}$ for a range of masses.

As will be seen from the above table, the values which Jeans suggests as a pure guess * for $M / M_{s}$ and $E^{\prime}-2$ and .00055 - are not compatible with each other. The interesting point which emerges, however, is that unless the numerical results of the equilibrium theory are very wide of the mark owing to the essential factors being ignored, a lower limit for the mass of Van Maanen's Star can be roughly estimated. The mean density cannot be greater than the calculated limiting density, and it is probably several times smaller.

* J. H. Jeans, ‘The Universe Around Us,' p. 310 (C. U. P. 1929). 


\author{
Equilibrium of Dense Stars.
}

959

From this it is possible to draw the following conclusions for Van Maanen's Star :-

$$
\mathrm{M} / \mathrm{M}_{s}>\cdot 4, \quad \rho>650,000, \quad \mathrm{E}^{\prime}<\cdot 0008 .
$$

For Procyon B the spectral type is unknown, though it is probably $\mathrm{M}^{\text {or }} \mathrm{K}^{*}$. It is, however, of interest to examine how far the star approaches the condensed type if a reasonable value of the surface temperature is assumed. For assumed temperatures of 3000 ( $\mathrm{M}$ type) and 4000 ( $\mathrm{K}$ type) the estimated correction $\dagger$ to convert $M_{\text {vis. }}$ to $M_{\text {bol. }}$ may be applied, and using (27), (29), and (30), the following results are obtained :-

\begin{tabular}{|c|c|c|c|c|}
\hline \multicolumn{5}{|c|}{$\begin{array}{l}T_{A B L E} V I \\
\text { Values for }\end{array}$} \\
\hline & $\mathrm{M} / \mathrm{M}_{s}=\cdot 37$ & & $=\cdot 84 \times$ & \\
\hline $\begin{array}{c}\mathrm{T}_{e} \\
3000\end{array}$ & $\begin{array}{c}\mathbf{M}_{\text {bol. }} \\
14\end{array}$ & $\begin{array}{c}R . \\
056\end{array}$ & $\begin{array}{c}\rho . \\
24 \times 10^{3}\end{array}$ & $\begin{array}{l}E^{\prime} . \\
.001\end{array}$ \\
\hline 4000 & $15 \%$ & .018 & $1 \cdot 1 \times 10^{5}$ & .0003 \\
\hline
\end{tabular}

Of course nothing definite can be deduced from these resulta, as in the case of Van Maanen's Star. They simply serve to show that unless the surface temperature is considerably greater than 3000 , Procyon $\mathrm{B}$ does not approach the limiting condensed state, the mean density being much smaller than that of other white dwarts.

\title{
Generation of Energy.
}

Some of the more important observed and deduced data for the three white dwarfs about which most is known are collected together, for convenience in comparison, in Table VII. The total energy radiated per second is symbolized by $\mathrm{E}$, the energy per gram by $\mathrm{E}^{\prime}$.

As was to be expected, the calculated limiting density $\rho_{0}$ is considerably greater than the "observed" mean density. The polytrope ratios (Table IV.) suggest that the maximum mean density may be something approaching 50 times smaller than $\rho_{0}$ for Sirius $B$, and 6 times smaller for

* J. H. Jeans, 'Astronomy and Cosmogony', p. 128.

$\uparrow$ Ibid. p. 46. 
1

2

3

4

5

6

7

8

9

10

11

12

13

14

15

16

17

18

19

\section{Dr. E. C. Stoner on the}

$\mathrm{O}_{2}$ Eridani B. If these ratios are approximately correct, $o_{2}$ Eridani $\mathrm{B}$ may be said to approach the limiting condensed state fairly closely, while Sirius B is still far removed from it. If the observational data (Table II.) and the essentials of the equilibrium theory are correct, Van Maanen's Star is certainly the most dense that is known, though it is impossible to saly how nearly it approaches the condensed limit.

If the observational data are correct, again, there is for stars of this type no simple relation between the mass and luninosity - as is shown by the absolute bolometric magnitude of $o_{2}$ Eridani B being smaller than that of Sirius B, although its mass is smaller. The radiation per gram, moreover, does not decrease steadily with the mass of the star, as had been suggested *.

TABLE VII.

Observed and Deluced Data for White Dwarfs.

\begin{tabular}{|c|c|c|c|c|c|c|c|}
\hline \multirow{2}{*}{-} & \multirow{2}{*}{$\mathrm{MI} / \mathrm{M}_{s}$} & \multirow{2}{*}{$\overline{\mathrm{T}_{\rho}}$} & & $-\overline{-}$ & \\
\hline & & & $\mathrm{R}$. & $n$ & $\rho_{41}$ calce. & 1. & $\mathrm{E}^{\prime}$ \\
\hline Sirius $B$ & 8.5 & 8,000 & 0312 & 40,000 & $23 \times 10^{6}$ & $1 \cdot 1 \times 10^{31}$ & 0065 \\
\hline$o_{2}$ Eridani $B \ldots \ldots \ldots$ & $\cdot 44$ & 11,200 & .3187 & $95,00 n$ & $14 \times 10^{6}$ & $1 f \times 10^{31}$ & .0185 \\
\hline Van M tanen's Star . & $>\cdot 4$ & $\tau, 000$ & 0095 & $>651), 000$ & $>1.1 \times 10^{6}$ & $64 \times 10^{29}$ & $<\cdot 0008$ \\
\hline
\end{tabular}

According to Jeans's hypothesis, which, in spite of its speculative nature, certainly satisfies most of the conditions, the energy generation in stars is to be traced to electronproton annihilation occurring in hyper-uranium atoms as a result of one of the extra-nuclear electrons of the atom falling into the nucleus. In completely ionized stars of the white dwarf type this process could not occur, so that the small radiation per gram of these stars is in complete accordance with the hypothesis. The radiation would be mainly due to the atoms in the outer layers of the star, so that there is not necessarily any simple relation between either the mass or the mean density and the amount of radiation per gram. It does none the less remain peculiar that $o_{2}$ Eriduni $B$, which approaches the condensed state, should bave a much higher surface temperature than Sirius B, and that it shoul.l generate more energy per gram. The material available in this connexion, however, is too slight to permit of any inductive generalizations being mude.

* J. H. Jeans, 'The Universe Around Us,' p. 310 . 


\section{The Fermi Statistics of Electron Gas at Lower Densities.}

The densities of white dwarfs are very much greater than those of other stars, in which the densities are too low and the temperatures too high for the electron gas to approach the condensed state. According to Jeans's theory of stellar evolution (outlined in I.), the tenanted portions of the Russell temperature-luminosity diagram correspond to stable states in which the pressures exceed considerably the perfect gaspressures. There will be a range of stability for each successive stage of ionization of the atoms, and this, on the liquid-star hypothesis, corresponds to the "jamming" of the incompletely-ionized atoms. The difficulty about this hypothesis is that the size which must be attributed to the atoms is very much greater than that indicated by other evidence. Now, with the Fermi statistics the pressure is always greater than that of a perfect gas, and it is interesting to note that there will be a maximum deviation corresponding to each stage of ionization. When with increasing temperature there is an increase in the number of electrons through ionization, there will be a deviation from the perfect gas relation between pressure and number of molecules. This deviation will decrease as the temperature increases, and will increase again when the next stage of ionization will increase. The deviations required, however, are much greater than those indicated by the Fermi statistics. This may be illustrated by taking a particular case and making the most favourable estimates. To ensure stability at the centre of the star, according to .Jeans, the pressure must be at least $1 \cdot 107$ times the normal pressure*. The pressure of electron gas is given by

$$
p=n l \mathrm{~T}\left\{1+\frac{1}{16 \times 2} \frac{n h^{3}}{(\pi m \bar{T})^{3 / 2}} \ldots\right\} .
$$

Inserting numerical values

$$
p=n k \mathrm{~T}\left\{1+3.62 \times 10^{-17} \frac{n}{\mathrm{~T}^{3 / 2}} \ldots\right\} . .
$$

Taking $\rho$ as $2.5 n m_{\mathrm{H}}$, Table VIII. gives the corresponding values of $\mathrm{T}, n$, and $\rho$ for the pressure to exceed the normal by 10 per cent.

In stars generally, for a given central temperature the central density is of order of ten times less than that in

* J. H. Jeans, 'Astronomy and Cosmogony,' p. 141.

Phil. Mag. S. 7. Vol.9. No.60. May $1930 . \quad 3$ R 
the table. For the sun ${ }^{*}$ the central density is about 140 , and the temperature can hardly be less than $40 \times 10^{6}$. This gives

$$
p=n k \mathrm{~T}\left(1+0.48 \times 10^{-2}\right) . . \quad . \quad .
$$

The pressure is thus about $\frac{1}{2}$ per cent. ahove normal instead of the required 10 per cent. It wonld seem, therefore, that unless there are other parts of the theory requiring modification, a simple application of the Fermi statistics is unable to remove the difficulties, although it does lead to deviations from the perfect gas laws of the required type.

\section{Table VIII.}

Corresponding Values for the Pressure to exceed normal by 10 per cent.

\begin{tabular}{|c|c|c|}
\hline $\mathrm{T}$ & $n$. & $\rho$. \\
\hline $10^{2}$ & $276 \times 10^{2 \times}$ & $1.145 \times 10^{-5}$ \\
\hline $30^{4}$ & $2 \cdot 7 t \times 10^{21}$ & $1 \cdot 145 \times 10^{-2}$ \\
\hline $10^{5}$ & $2 \cdot 76 \times 10^{24}$ & $1 \cdot 14 i \times 10$ \\
\hline $10^{x}$ & $276 \times 10^{27}$ & $1.145 \times 10^{4}$ \\
\hline
\end{tabular}

\section{Conclusion.}

It is generally agreed that the dense dwarf stars represent an advanced stage of stellar evolution. It is reasonable to suppose that the white dwarf stage, corresponding to high surface temperature, will be a comparatively short one, and since the stars are small, it is probable that most condensed stars are too faint to be observed. From the mass luminosity relation, moreover, even treating this as a purely empirical generalization, the masses of condensed stars will in general be small. There is therefore a strong probability that stars of the type in which the gravitational kinetic equilibrium discussed in this paper is of importance are much more numerous than is suggested by the number of known examples. For these the theory may be said to account in a general way for the order of magnitude of the densities deduced from the observational data.

For more normal stars the application of the Fermi statistics shows that there will be an appreciable deviation from the perfect gas laws for the electron gas. Although the deviations will be of the kind required in Jeans's theory

* Ibid.p. 104. 


\section{Equilibrium of Dense Stars.}

of stability, they are not nearly large enough. It would seem, therefore, either that the theory as to the conditions for stability requires modification, or that there are factors involved which are at present unrecognized.

\section{Summary.}

In a previous paper the conclusion was reached that there was a limiting density for stars in which the atoms were completely ionized, varying as the square of the mass of the star. The limiting state occurs when the decrease in gravitational energy on contraction is equal to the increase in the total kinetic energy of the electron gas. In the treatment the relativity change of mass with velocity was neglected. Some approximate calculations by Anderson indicate the general effect of this change of mass, which necessitates a modification of the previous conclusions when the mass of the star becomes comparable with that of the sun.

In the present paper the effect of the relativity change of mass is worked out with more rigour for the idealized case for a sphere of uniform density. For spheres of increasing mass the limiting density varies at first as the square of tho mass, and then more rapidly, there being a limiting mass $\left(2.19 \times 10^{33}\right)$ above which the gravitational kinetic equilibrium considered will not occur. Tables and curves are given showing the relation between mass and limiting density.

It is shown that the distribution of density in condensed stars will be polytropic, and rough estimates are made of the ratio of the central to the mean density.

The observational and deduced data for known white dwarfs are considered in some detail. The density of Sirius B is well below the limit calculated, while that of $o_{2}$ Eridani $\mathbf{B}$ approaches it. The theory enables a lower limit, for the mass and density of Van Maanen's Star to be roughly estimated. The observational, deduced, and calculated data for these three stars, and also for Procyon B, are given in tables.

The deduced data indicate that the generation of energy per gram in known dense stars is not simply related to the mass of the star.

The application of the Fermi statisties to the electron gas in normal stars is briefly discussed in connexion with Jeans's theory of stability. There will be deviations from the perfect gas laws of the type required, but they are nbout twenty times too small to satisfy the stability conditions.

Physics Department,

The University, Leeds.

December 1929.

$3 \mathrm{R} 2$

http://mc.manuscriptcentral.com/pm-pml 